\title{
ON $p$-ELLIPTIC DIVERGENCE FORM OPERATORS AND HOLOMORPHIC SEMIGROUPS
}

\author{
MORITZ EGERT
}

\begin{abstract}
Second order divergence form operators are studied on an open set with various boundary conditions. It is shown that the $p$-ellipticity condition of CarbonaroDragičević and Dindoš-Pipher implies extrapolation to a holomorphic semigroup on Lebesgue spaces in a $p$-dependent range of exponents that extends the maximal range for general strictly elliptic coefficients. This has immediate consequences for the harmonic analysis of such operators, including $\mathrm{H}^{\infty}$-calculi and Riesz transforms.
\end{abstract}

\section{INTRODUCTION AND MAIN RESUltS}

Let $A: O \rightarrow \mathcal{L}\left(\mathbb{C}^{d}\right)$ be a measurable strictly elliptic matrix function on an open set $O \subseteq \mathbb{R}^{d}$, that is to say, there are constants $\lambda, \Lambda>0$ such that for almost every $x \in O$,

$$
|A(x) \xi| \leq \Lambda|\xi| \quad \text { and } \quad \operatorname{Re}(A(x) \xi \mid \xi) \geq \lambda|\xi|^{2}
$$

hold for all $\xi \in \mathbb{C}^{d}$. The associated divergence form operator $L=-\operatorname{div}(A \nabla \cdot)$ is defined on $\mathrm{L}^{2}(O)$ in the weak sense through a sesquilinear form

$$
a: V \times V \rightarrow \mathbb{C}, \quad a(u, v)=\int_{O} A \nabla u \cdot \overline{\nabla v} \mathrm{~d} x,
$$

where (mixed) Dirichlet and Neumann boundary conditions are incorporated through the choice of the form domain $\mathrm{W}_{0}^{1,2}(O) \subseteq V \subseteq \mathrm{W}^{1,2}(O)$, see Section 3 below. It is well known that $L$ is maximal accretive $[18$, VI.6] and therefore $-L$ generates a holomorphic $C_{0}$-semigroup of contractions $T=(T(t))_{t \geq 0}$ on $\mathrm{L}^{2}(O)$ of angle $\pi / 2-\omega$, where

$$
\omega:=\sup _{u \in V} \arg a(u, u),
$$

see [18, XI.6, Thm. 1.24]. In this paper, we are concerned with extrapolating $T$ by density to a holomorphic $C_{0}$-semigroup on $\mathrm{L}^{q}(O)$ for $q$ in an interval around 2 .

For real coefficient matrices, it has been long known that $T$ extrapolates to $\mathrm{L}^{q}(O)$ for all $q \in[1, \infty)$, see, for example, $[1,13,21]$. For complex matrices, on the contrary, there is a natural threshold in $q$ that is related to Sobolev embeddings. For $|1 / 2-1 / q|<$ $1 / d$ extrapolation is well-known by various different proofs on various classes of open sets $[3,7,9,21,23]$. In the plane this covers the full range $q \in(1, \infty)$, but in dimensions $d \geq 3$ the semigroup may cease from extrapolating if $|1 / 2-1 / q|>1 / d$, even on $O=\mathbb{R}^{d}$, see [15, Prop. 2.10].

Date: February 18, 2021.

2010 Mathematics Subject Classification. 47D06, 35J15, 47B44.

Key words and phrases. divergence form operators on open sets, $p$-ellipticity, holomorphic semigroups, dissipative operators, ultracontractivity, off-diagonal estimates. 
In their groundbreaking paper [5], Maz'ya and Cialdea have considered operators with coefficients $A \in \mathrm{C}^{1}\left(\bar{O} \rightarrow \mathcal{L}\left(\mathbb{C}^{d}\right)\right)$ and pure Dirichlet boundary conditions on a bounded regular domain. They have found an algebraic condition on the matrix $A$ that is sufficient for $T$ to extrapolate to a contraction semigroup on $\mathrm{L}^{p}(O)$ and that is also necessary for the latter to hold if, in addition, $\operatorname{Im}(A)$ is symmetric. This was generalized to the setup described above, but still for pure Dirichlet boundary conditions, by Carbonaro and Dragičević [4]. They have elegantly rephrased the condition in [5] as

$$
\Delta_{p}(A):=\operatorname{essinf}_{x \in O} \min _{\xi \in \mathbb{C}^{d},|\xi|=1} \operatorname{Re}\left(A(x) \xi, \mathcal{J}_{p} \xi\right) \geq 0,
$$

where $\mathcal{J}_{p}: \mathbb{C}^{d} \rightarrow \mathbb{C}^{d}$ is the $\mathbb{R}$-linear map defined by

$$
\mathcal{J}_{p}(\alpha+\mathrm{i} \beta)=2\left(\frac{\alpha}{p^{\prime}}+\frac{\mathrm{i} \beta}{p}\right) \quad\left(\alpha, \beta \in \mathbb{R}^{d}\right)
$$

and $p^{\prime}=p /(p-1)$ is the Hölder conjugate of $p \in(1, \infty)$. The stronger condition $\Delta_{p}(A)>$ 0 was also introduced in [4] in the context of dimension-free bilinear embeddings and independently by Dindoš-Pipher [8] in the context of boundary value problems. We shall follow terminology of [4] and say that $A$ is $p$-elliptic in this case. By definition, this means for almost every $x \in O$ the $p$-adapted lower bound

$$
\operatorname{Re}\left(A(x) \xi, \mathcal{J}_{p} \xi\right) \geq \Delta_{p}(A)|\xi|^{2} \quad \text { with } \quad \Delta_{p}(A)>0
$$

for all $\xi \in \mathbb{C}^{d}$. This can be viewed as an interpolating condition between general strictly elliptic and strictly elliptic real matrices. Indeed, (1) automatically implies $\Delta_{2}(A) \geq \lambda$ and if in addition $A$ is real, then we have $\Delta_{p}(A) \geq \lambda\left(2 / p^{\prime} \wedge 2 / p\right)$ for every $p \in(1, \infty)$.

Throughout the paper, as far as $p$-ellipticity is concerned, we assume $p \in(1, \infty)$. This will imply that all appearing Lebesgue exponents $q$ also belong to this open interval.

1.1. Main results. Our main result gives an extension of the extrapolation range for operators with $p$-elliptic coefficients that bridges the gap between the optimal ranges for general complex and real coefficients. In view of the discussion above this is only of interest in dimension $d \geq 3$. What remains open is the question whether this range is optimal over the class of all $p$-elliptic matrices.

We shall work under the assumption of (Sobolev) embedding properties of the form domain $V$ that are made precise in Section 3.2. Here, we only mention that the homogeneous version holds without any restrictions on $O$ in case of pure Dirichlet conditions and for mixed boundary conditions if $O$ is bounded, connected, and Lipschitz regular around the Neumann boundary part.

Theorem 1. Let $d \geq 3$ and assume that $V$ has the embedding property. If $A$ is p-elliptic, then for every $\varepsilon>0$ the semigroup $\left(\mathrm{e}^{-\varepsilon t} T(t)\right)_{t \geq 0}$ generated by $-L-\varepsilon$ extrapolates to a $C_{0}$-semigroup on $\mathrm{L}^{q}(O)$ provided that

$$
|1 / 2-1 / q| \leq 1 / d+(1-2 / d)|1 / 2-1 / p| .
$$

This semigroup is bounded holomorphic of angle $\pi / 2-\omega$. If $V$ has the homogeneous embedding property, then the same result also holds for $\varepsilon=0$. 
We adopt the notion of bounded holomorphic semigroups from [14]. Uniform boundedness is required on every sector of angle $\psi<\pi / 2-\omega$ but with possibly $\psi$-dependent bound. The extrapolation to a $C_{0}$-semigroup in Theorem 1 was known previously only under the structural assumption that $\operatorname{Im} A$ is symmetric [11, Thm. 1.4]. See also [11,17,20,21] for earlier contributions. We remark that Theorem 1 applies in particular to $q=p$ and $q=p^{\prime}$.

The proof will be given at the end of Section 5. It follows a two-step procedure: If we can extrapolate the semigroup to $\mathrm{L}^{p}(O)$, then we can use ultracontractivity to extrapolate further to the range of $q$ 's in Theorem 1. An essential tool in this approach are $\mathrm{L}^{2}$ offdiagonal bounds for $T$ that we reproduce in Section 3.3 for convenience. The required extrapolation to $\mathrm{L}^{p}(O)$ in turn relies on some of the fundamental algebraic calculations in [5]. In fact, we obtain as our second main result in Section 4, and without any further assumptions on $V$ besides its mere definition at the start of Section 3, the following

Theorem 2. If $\Delta_{p}(A) \geq 0$, then $T$ extrapolates to a holomorphic $C_{0}$-semigroup of contractions on $\mathrm{L}^{q}(O)$ provided that $|1 / 2-1 / q|<|1 / 2-1 / p|$. If $A$ is $p$-elliptic, then these properties also hold at the endpoints $q=p$ and $q=p^{\prime}$.

By this means, we generalize the implication " $(a) \Rightarrow(b)$ " in $[4$, Thm. 1.3] to more general boundary conditions.

1.2. Consequences. Let us recall the important observation of [4, Prop. 5.15] that $p$ ellipticity of $A$ can equivalently be stated through the inequality

$$
\mu(A):=\operatorname{essinf}_{x \in O} \inf _{\xi \in \mathbb{C}^{d},(A(x) \xi \mid \bar{\xi}) \neq 0} \frac{\operatorname{Re}(A(x) \xi \mid \xi)}{|(A(x) \xi \mid \bar{\xi})|}>|1-2 / p|,
$$

which decouples $p$ and $A$. For convenience, we have included a direct proof in appendix. We conclude that the set $\{p: A$ is $p$-elliptic $\}$ is open in $(1, \infty)$. This being said, our main results reveal some new features even for the classical strictly elliptic matrices as in (1). Indeed, we obtain from the trivial bound $\mu(A) \geq \lambda / \Lambda$ that every strictly elliptic matrix is $p$-elliptic provided that $|1 / 2-1 / p|<\lambda /(2 \Lambda)$ and we conclude from Theorem 1 the following

Corollary 3. Let $d \geq 3$ and assume that $V$ has the homogeneous embedding property. Then $T$ extrapolates to a bounded holomorphic $C_{0}$-semigroup on $\mathrm{L}^{q}(O)$ of angle $\pi / 2-\omega$ provided that

$$
|1 / 2-1 / q|<1 / d+(1 / 2-1 / d) \lambda / \Lambda
$$

Extrapolation beyond the range $|1 / 2-1 / q| \leq 1 / d$ is essentially known in this context. Our proof here, however, appears particularly clean in that it avoids the sophisticated selfimprovement properties of invertibility in complex interpolation scales [3,9] or reverse Hölder inequalities [23] and, as a consequence, pinpoints the improvement in terms of ellipticity.

An independent interest in Theorem 1 stems from its immediate consequences for the harmonic analysis of $L$ on $\mathrm{L}^{q}(O)$, such as $\mathrm{H}^{\infty}$-calculus, Riesz transforms and Kato square root estimates, at least when $O$ is either the whole space or a bounded connected set that 
is Lipschitz regular around the Neumann boundary part. Indeed, such results come for free once the extrapolation of the semigroup has been settled and we refer the reader to $[3,9]$ for a precise account.

Acknowledgments. We are grateful to Andrea Carbonaro for inspiring discussions during a workshop at CIRM in April 2018 and to Oliver Dragičević for sharing further insight on and around the topic. We acknowledge that a result similar to our Theorem 1 has been discovered independently in [12] by ter Elst, Haller-Dintelmann, Rehberg, and Tolksdorf through a different method. This research has been supported by the ANR project RAGE ANR-18-CE40-0012-01.

\section{Notation}

We use $|\cdot|$ for both the Euclidean norm on $\mathbb{C}^{d}, d \geq 1$, and the operator norm of matrices viewed as linear operators on the Hilbert space $\mathbb{C}^{d}$. Inner products $(\cdot \mid \cdot)$ on complex Hilbert spaces are linear in the first component. We use the same symbol for the $\mathrm{L}^{p}-\mathrm{L}^{p^{\prime}}$ duality pairing that extends the inner product on $\mathrm{L}^{2}$. We denote the (semi)-distance between subsets $E, F$ of $\mathbb{R}^{d}$ by $\mathrm{d}(E, F)$. Given $z \in \mathbb{C}$, we write

$$
\operatorname{sgn} z= \begin{cases}z /|z| & \text { if } z \neq 0, \\ 0 & \text { if } z=0 .\end{cases}
$$

For $\psi \in(0, \pi)$, we define the open sector $\mathrm{S}_{\psi}^{+}:=\{z \in \mathbb{C} \backslash\{0\}:|\arg (z)|<\psi\}$ of opening angle $2 \psi$ symmetric about $(0, \infty)$ and for convenience we put $\mathrm{S}_{0}^{+}:=(0, \infty)$. Occasionally, we use the symbol $\lesssim$ for inequalities that hold up to multiplication by a constant. None of our constants depends on the structure of $A$ itself but only on quantified parameters such as $\lambda, \Lambda, \Delta_{p}(A)$.

All function spaces in this paper are over the complex numbers. By $\mathrm{W}^{1,2}(O)$, we denote the usual Sobolev space of functions $u \in \mathrm{L}^{2}(O)$ with distributional gradient $\nabla u \in \mathrm{L}^{2}(O)^{d}$ equipped with the Hilbertian norm $\|u\|_{1,2}:=\left(\|u\|_{2}^{2}+\|\nabla u\|_{2}^{2}\right)^{1 / 2}$. Given a closed set $D \subseteq \partial O$, we define the class of test functions that vanish in a neighborhood of $D$,

$$
\mathrm{C}_{D}^{\infty}(O):=\left.\mathrm{C}_{0}^{\infty}\left(\mathbb{R}^{d} \backslash D\right)\right|_{O},
$$

and we let $\mathrm{W}_{D}^{1,2}(O)$ be its closure in $\mathrm{W}^{1,2}(O)$. We have $\mathrm{W}_{\partial O}^{1,2}(O)=\mathrm{W}_{0}^{1,2}(O)$ but on an arbitrary open set the space obtained for $D=\emptyset$ can be a proper subspace of $\mathrm{W}^{1,2}(O)$.

\section{THE FORM DOMAIN $V$}

We shall always assume that the form domain $V$ in (2) is one of the following closed subspaces of $\mathrm{W}^{1,2}(O)$ :

- $V=\mathrm{W}^{1,2}(O)$ corresponding to Neumann boundary conditions for $L$ or

- $V=\mathrm{W}_{D}^{1,2}(O)$ for a closed set $D \subseteq \partial O$, corresponding to mixed boundary conditions or, more precisely, Dirichlet conditions on $D$ and Neumann conditions on $\partial O \backslash D$.

The second case includes pure Dirichlet boundary conditions $(D=\partial O)$ and what is usually called "good Neumann" boundary conditions $(D=\emptyset)$. In this section, we 
prove important invariance properties for $V$ and outline their consequences for the semigroup $T$.

3.1. Invariance properties. We shall frequently use the classical result [21, Prop. 4.4] that for all $u \in \mathrm{W}^{1,2}(O)$ we have $|u| \in \mathrm{W}^{1,2}(O)$ with

$$
\nabla|u|=\operatorname{Re}(\overline{\operatorname{sgn}(u)} \nabla u) .
$$

For real-valued $u$, this can be seen as a particular instance of the chain rule, which holds more generally for the composition $\Phi \circ u$ of a real-valued $u \in \mathrm{W}_{\text {loc }}^{1,1}(O)$ with a Lipschitz function $\Phi: \mathbb{R} \rightarrow \mathbb{R}$, see, for example, [24, Thm. 2.1.11]. We would like to draw the reader's attention to the particularity that the chain rule does not hold in the same generality for functions $u$ valued in $\mathbb{C} \cong \mathbb{R}^{2}$ and Lipschitz functions $\Phi: \mathbb{C} \rightarrow \mathbb{C}$, see [16]. What continues to hold for complex valued functions, though, is the bound

$$
|\nabla(\Phi \circ u)(x)| \leq \operatorname{Lip}(\Phi)|\nabla u(x)|
$$

for almost every $x \in O$. This follows immediately on approximating $u$ by smooth functions in $\mathrm{W}_{\text {loc }}^{1,1}(O), \Phi$ by smooth functions uniformly on $\mathbb{C}$, and using the ordinary chain rule for smooth functions.

Lemma 4 (Invariance properties). $\quad$ (i) If $\Phi: \mathbb{C} \rightarrow \mathbb{C}$ is Lipschitz continuous and satisfies $\Phi(0)=0$, then $\Phi \circ u \in V$ for all $u \in V$.

(ii) If $\varphi: \mathbb{R}^{d} \rightarrow \mathbb{C}$ is Lipschitz continuous and bounded, then $\varphi u \in V$ for all $u \in V$.

Proof. As for (i), we first note that (8) and the pointwise bound $|\Phi(z)| \leq \operatorname{Lip}(\Phi)|z|$ imply $\|\Phi \circ u\|_{1,2} \leq \operatorname{Lip}(\Phi)\|u\|_{1,2}$. If $V=\mathrm{W}^{1,2}(O)$, then we are done.

Otherwise, we have $V=\mathrm{W}_{D}^{1,2}(O)$ for some closed $D \subseteq \partial O$. By definition, there is a sequence $\left(u_{n}\right)_{n} \subseteq \mathrm{C}_{0}^{\infty}\left(\mathbb{R}^{d} \backslash D\right)$ such that $\left.u_{n}\right|_{O} \rightarrow u$ in $\mathrm{W}^{1,2}(O)$ as $n \rightarrow \infty$. We set $v_{n}:=\Phi \circ u_{n}$. Then $v_{n}$ is Lipschitz continuous and thanks to $\Phi(0)=0$ the properties of having compact support and vanishing in a neighborhood of $D$ carry over from $u_{n}$ to $v_{n}$. We conclude $v_{n} \in V$ since the required approximants in $\mathrm{C}_{0}^{\infty}\left(\mathbb{R}^{d} \backslash D\right)$ can explicitly be constructed by convolution with smooth, compactly supported kernels. The first part of the proof shows that $\left(v_{n}\right)_{n}$ is bounded in $V$. Hence, it admits a subsequence with weak limit $v_{\infty} \in V$. On the other hand, we have $\left\|v_{n}-\Phi \circ u\right\|_{2} \leq \operatorname{Lip}(\Phi)\left\|u_{n}-u\right\|_{2}$, so that $v_{n} \rightarrow \Phi \circ u$ strongly in $\mathrm{L}^{2}(O)$ as $n \rightarrow \infty$. Thus, we have $\Phi \circ u=v_{\infty} \in V$ as required.

As for (ii), we obtain $\|\varphi u\|_{1,2} \leq\left(\|\varphi\|_{\infty}+\|\nabla \varphi\|_{\infty}\right)\|u\|_{1,2}$ from the product rule and the rest of the proof follows the pattern of (i).

3.2. Embedding properties. These properties will only be relevant in dimension $d \geq 3$, in which case we denote by $2^{*}:=2 d /(d-2)$ the Sobolev conjugate of 2 .

Definition 5. Let $d \geq 3$. If $\|v\|_{2^{*}} \lesssim\|v\|_{1,2}$ holds for all $v \in V$, then $V$ has the embedding property. It has the homogeneous embedding property if $\|v\|_{2^{*}} \lesssim\|\nabla v\|_{2}$ holds for all for all $v \in V$. 
The space $V=\mathrm{W}_{0}^{1,2}(O)$ always has the homogeneous embedding property [24, Thm. 2.4.1]. A convenient way to approach more general $V$ is by means of a bounded extension operator $E: V \rightarrow \mathrm{W}^{1,2}\left(\mathbb{R}^{d}\right)$. Such operator exists, for example, under the assumption that $O$ is bounded and $\partial O$ admits Lipschitz coordinate charts around the Neumann boundary part [10, Sec. 6$]$. The embedding property then follows from the commutative diagram

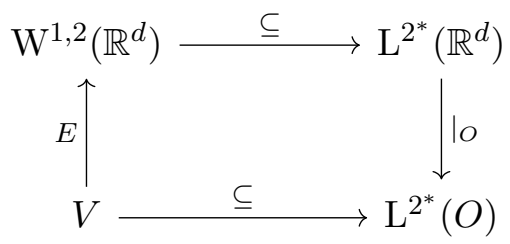

If $O$ is bounded and connected, then the embedding property in case of mixed boundary conditions already entails the seemingly stronger homogeneous version. This is due to the following lemma, the proof of which relies on an idea that we found in [6, Sec. 7]

Lemma 6. Let $d \geq 3$ and $V=\mathrm{W}_{D}^{1,2}(O)$ for a closed set $D \subseteq \partial O$. Assume $O$ is bounded, connected, and that $V$ has the embedding property. Then either $V$ has the homogeneous embedding property or $V=\mathrm{W}_{\emptyset}^{1,2}(O)$ models in fact (good) Neumann boundary conditions.

Proof. Step 1: $V \subseteq \mathrm{L}^{2}(O)$ is compact. Suppose $\left(v_{n}\right)_{n} \subseteq V$ converges to 0 weakly in $V$. We have to prove that it converges strongly to 0 in $\mathrm{L}^{2}(O)$. For any $\varphi \in \mathrm{C}_{0}^{\infty}(O)$ with $\|\varphi\|_{\infty} \leq 1$, we can use Hölder's inequality on $(1-\varphi) v_{n}$ and the embedding property for $V$ to give

$$
\left\|v_{n}\right\|_{2} \leq\left\|\varphi v_{n}\right\|_{2}+\left\|(1-\varphi) v_{n}\right\|_{2} \lesssim\left\|v_{n}\right\|_{2}+|\{x \in O: \varphi \neq 1\}|^{1 / d}\left\|v_{n}\right\|_{1,2} .
$$

Since $O$ is a bounded set and $\left(v_{n}\right)_{n}$ is bounded in $V$, the second term on the right can be made as small as we want through the choice of $\varphi$. Once $\varphi$ is fixed, we note that the first term tends to 0 as $n \rightarrow \infty$ since multiplication by $\varphi$ is a bounded operator $V \rightarrow \mathrm{W}_{0}^{1,2}(O)$ and the latter compactly embeds into $\mathrm{L}^{2}(O)$, see [24, Thm. 2.5.1].

Step 2: Poincaré alternative. If we have a Poincaré inequality $\|v\|_{2} \lesssim\|\nabla v\|_{2}$ for all $v \in V$, then the embedding property trivially implies the homogeneous one.

Now assume that this was not the case. Then there exists for every $n \in \mathbb{N}$ some $v_{n} \in V$ such that $\left\|v_{n}\right\|_{2}=1$ but $\left\|\nabla v_{n}\right\|_{2} \leq 1 / n$. Since $\left(v_{n}\right)_{n}$ is bounded, it admits a subsequence denoted again by $\left(v_{n}\right)_{n}$ that converges weakly in $V$ to some $v_{\infty} \in V$. Since $\nabla: V \rightarrow$ $\mathrm{L}^{2}(O)^{d}$ is bounded, we have $\nabla v_{n} \rightarrow \nabla v_{\infty}$ weakly in $\mathrm{L}^{2}(O)^{d}$ as $n \rightarrow \infty$. At the same time, this sequence converges strongly to 0 , meaning that $\nabla v_{\infty}=0$. Since $O$ is connected, we conclude that $v_{\infty} \in V$ is constant. By compactness of the embedding $V \subseteq \mathrm{L}^{2}(O)$ there is another subsequence that converges to $v_{\infty}$ strongly in $\mathrm{L}^{2}(O)$ and we conclude $\left\|v_{\infty}\right\|_{2}=1$.

So far we know that $V$ contains a nonzero constant function. Since $V$ is invariant under multiplication with $\mathrm{C}_{0}^{\infty}\left(\mathbb{R}^{d}\right)$ functions, see Lemma 4 , we conclude that it contains $\mathrm{C}_{\emptyset}^{\infty}(O)=\left.\mathrm{C}_{0}^{\infty}\left(\mathbb{R}^{d}\right)\right|_{O}$. Since $V$ is closed for the $\mathrm{W}^{1,2}(O)$ norm, we obtain $\mathrm{W}_{\emptyset}^{1,2} \subseteq V$. But the reverse inclusion holds by definition of $V$, and hence we have $\mathrm{W}_{\emptyset}^{1,2}=V$. 
3.3. Off-diagonal estimates. Lemma 4.(ii) along with Davies' perturbation method readily yields the following off-diagonal estimates for the semigroup $T$. For convenience, we include the short argument and give explicit constants.

Proposition 7. Let $\psi \in[0, \pi / 2-\omega)$. For all measurable sets $E, F \subseteq O$, all $z \in \mathrm{S}_{\psi}^{+}$, and all $f \in \mathrm{L}^{2}(O)$ with support in $E$ it follows

$$
\|T(z) f\|_{\mathrm{L}^{2}(F)} \leq \mathrm{e}^{-\frac{\mathrm{d}(E, F)^{2}}{4 C|z|}}\|f\|_{\mathrm{L}^{2}(E)}
$$

where $C=\Lambda+\left(\Lambda^{2} \cos (\omega)\right) /(\lambda \cos (\psi+\omega))$.

Proof. We begin with off-diagonal bounds for $z=t>0$. Let $\varphi: \mathbb{R}^{d} \rightarrow \mathbb{R}$ be bounded and Lipschitz continuous with $\|\nabla \varphi\|_{\infty} \leq 1$ and let $\rho>0$; both yet to be specified. Then $\mathrm{e}^{ \pm \rho \varphi}$ are also bounded and Lipschitz continuous. Since according to Lemma 4 the form domain $V$ is invariant under multiplication with $\mathrm{e}^{ \pm \rho \varphi}$, we can define $\mathrm{e}^{\rho \varphi} L \mathrm{e}^{-\rho \varphi}$ by means of the sesquilinear form

$$
b: V \times V \rightarrow \mathbb{C}, \quad b(u, v)=a\left(\mathrm{e}^{-\rho \varphi} u, \mathrm{e}^{\rho \varphi} v\right) .
$$

We multiply out the expression for $a\left(\mathrm{e}^{-\rho \varphi} u, \mathrm{e}^{\rho \varphi} u\right)$ and then use the ellipticity estimates $|a(u, u)| \leq \Lambda\|\nabla u\|_{2}^{2}$, Re $a(u, u) \geq \lambda\|\nabla u\|_{2}^{2}$, and Young's inequality with $\varepsilon \in(0,1)$ to give

$$
|b(u, u)| \leq 2 \Lambda\|\nabla u\|_{2}^{2}+2 \Lambda \rho^{2}\|u\|_{2}^{2}
$$

and

$$
\operatorname{Re} b(u, u) \geq(1-\varepsilon) \lambda\|\nabla u\|_{2}^{2}-\left(\Lambda+\frac{\Lambda^{2}}{\varepsilon \lambda}\right) \rho^{2}\|u\|_{2}^{2} .
$$

We put $C:=\Lambda+\Lambda^{2} /\left(\varepsilon^{2} \lambda\right)$ and conclude that $\mathrm{e}^{\rho \varphi} L \mathrm{e}^{-\rho \varphi}+C \rho^{2}$ is again a maximal accretive operator on $\mathrm{L}^{2}(O)$, and hence its negative generates a $C_{0}$-semigroup of contractions $(S(t))_{t \geq 0}$. In fact, this is the transformed semigroup $S(t)=\mathrm{e}^{-C \rho^{2} t} \mathrm{e}^{\rho \varphi} T(t) \mathrm{e}^{-\rho \varphi}$, see $\left[14\right.$, II.2.a]. Now, we specialize to $\varphi(x)=\mathrm{d}(x, E) \wedge n$ for some $n \in \mathbb{N}$. For $f \in \mathrm{L}^{2}(O)$ supported in $E$ we obtain

$$
T(t) f=\mathrm{e}^{C \rho^{2} t} \mathrm{e}^{-\rho \varphi} S(t) \mathrm{e}^{\rho \varphi} f=\mathrm{e}^{C \rho^{2} t} \mathrm{e}^{-\rho \varphi} S(t) f
$$

and consequently

$$
\|T(t) f\|_{\mathrm{L}^{2}(F)} \leq \mathrm{e}^{C \rho^{2} t-\rho(\mathrm{d}(E, F) \wedge n)}\|f\|_{\mathrm{L}^{2}(E)} .
$$

It remains to optimize the parameters $\rho, n$, and $C=C(\varepsilon)$. For $\rho=(\mathrm{d}(E, F) \wedge n) /(2 C t)$, the argument of the exponential function takes its global minimum $-(\mathrm{d}(E, F) \wedge n)^{2} /(4 C t)$. Then we pass to the limits as $n \rightarrow \infty$ and $\varepsilon \rightarrow 1$ and the claim for $z=t>0$ follows.

In the general case $z \in \mathrm{S}_{\psi}^{+}$, we replace $L$ by the operator $\mathrm{e}^{\mathrm{i} \arg z} L$. The associated sesquilinear form $\mathrm{e}^{\mathrm{i} \arg z} a(\cdot, \cdot)$ has the same upper bound $\Lambda$ as $a$ and, by rotation of the numerical range, we obtain the lower bound

$$
\min _{z^{\prime} \in \mathrm{S}_{\omega}^{+}, \operatorname{Re} z^{\prime} \geq \lambda} \operatorname{Re}\left(\mathrm{e}^{\mathrm{i} \arg z} z^{\prime}\right)=\lambda \frac{\cos (\psi+\omega)}{\cos (\omega)}>0 .
$$

The claim follows from the first part with $t=|z|$ on noting that $\mathrm{e}^{-z L}=\mathrm{e}^{-t \mathrm{e}^{\mathrm{i} \arg z} L}$. 


\section{L $^{p}$-DISSIPATIVITY}

Here, we use the algebraic properties of matrices that satisfy Maz'ya and Cialdea's condition $\Delta_{p}(A) \geq 0$ to prove that $L$ is formally $\mathrm{L}^{p}$-dissipative. From this, we shall conclude Theorem 2.

Some considerations will become less technical for $p \in[2, \infty)$. For the results we are after, this will not cause any burden because we can rely on duality and the following observation that already appears in [4, Prop. 5.8 \& Cor. 5.17.3]. We reproduce the easy proof in order to become acquainted with the definition of $\Delta_{p}(A)$ from (4).

Lemma 8. Let $p \in(1, \infty)$. If $A$ is $p$-elliptic, then both $A$ and $A^{*}$ are p-elliptic and $p^{\prime}$-elliptic. More precisely, for any $A$ it follows that

$$
\text { (i) } \quad \Delta_{p}(A)=\Delta_{p^{\prime}}(A), \quad \text { (ii) } \quad \Delta_{p}\left(A^{*}\right)=\Delta_{p^{\prime}}\left(A^{*}\right) \geq \Delta_{p}(A)\left(p / p^{\prime} \wedge p^{\prime} / p\right) .
$$

Proof. Let $\xi \in \mathbb{C}^{d}$ and $x \in O$. We have $\mathrm{i} \mathcal{J}_{p^{\prime}} \xi=\mathcal{J}_{p}(\mathrm{i} \xi)$, and hence $\left(A(x) \xi, \mathcal{J}_{p^{\prime}} \xi\right)=$ $\left(A(x) \mathrm{i} \xi, \mathcal{J}_{p}(\mathrm{i} \xi)\right)$. This yields (i) as well as the equality in (ii) when applied to $A^{*}$ instead of $A$. Next, we have $p p^{\prime} \mathcal{J}_{p} \mathcal{J}_{p^{\prime}} \xi=4 \xi$ and conclude that

$$
\operatorname{Re}\left(A^{*}(x) \xi \mid \mathcal{J}_{p^{\prime}} \xi\right)=\frac{p p^{\prime}}{4} \operatorname{Re}\left(A^{*}(x) \mathcal{J}_{p} \mathcal{J}_{p^{\prime}} \xi \mid \mathcal{J}_{p^{\prime}} \xi\right)=\frac{p p^{\prime}}{4} \operatorname{Re}\left(A(x) \mathcal{J}_{p^{\prime}} \xi \mid \mathcal{J}_{p} \mathcal{J}_{p^{\prime}} \xi\right) .
$$

To obtain the inequality in (ii), it suffices to remark that $\left|\mathcal{J}_{p^{\prime}} \xi\right|^{2} \geq 4\left(1 / p^{\prime} \wedge 1 / p\right)^{2}|\xi|^{2}$.

We continue with a purely algebraic calculation.

Lemma 9. Almost everywhere on $O$, the following holds for all $Z=X+\mathrm{i} Y, X, Y \in \mathbb{R}^{d}$ :

$$
\begin{aligned}
\operatorname{Re}(A Z \mid Z)- & \left(1-\frac{2}{p}\right)^{2} \operatorname{Re}(A X \mid X)-\left(1-\frac{2}{p}\right) \operatorname{Im}\left(\left(A-A^{*}\right) X \mid Y\right) \\
& \geq \Delta_{p}(A)\left(\frac{2}{p}|X|^{2}+\frac{p}{2}|Y|^{2}\right) .
\end{aligned}
$$

Proof. We have

$$
\operatorname{Re}(A Z \mid Z)=\operatorname{Re}(A X \mid X)+\operatorname{Re}(A Y \mid Y)+\operatorname{Im}\left(\left(A+A^{*}\right) X \mid Y\right)
$$

and in the same manner

$$
\frac{1}{2} \operatorname{Re}\left(A \widetilde{Z} \mid \mathcal{J}_{p} \widetilde{Z}\right)=\frac{1}{p^{\prime}} \operatorname{Re}(A \tilde{X} \mid \tilde{X})+\frac{1}{p} \operatorname{Re}(A \tilde{Y} \mid \tilde{Y})+\operatorname{Im}\left(\left(\frac{1}{p} A+\frac{1}{p^{\prime}} A^{*}\right) \tilde{X} \mid \tilde{Y}\right)
$$

for all $\tilde{X}, \tilde{Y} \in \mathbb{R}^{d}$ and $\widetilde{Z}=\tilde{X}+\mathrm{i} \tilde{Y}$. We apply the second equality to $\widetilde{X}=(2 / \sqrt{p}) X$ and $\widetilde{Y}=\sqrt{p} Y$, and then use the first equality to deduce

$$
\begin{aligned}
\frac{1}{2} \operatorname{Re}\left(A \widetilde{Z} \mid \mathcal{J}_{p} \widetilde{Z}\right)= & \frac{4}{p p^{\prime}} \operatorname{Re}(A X \mid X)+\operatorname{Re}(A Y \mid Y)+\operatorname{Im}\left(\left(\frac{2}{p} A+\frac{2}{p^{\prime}} A^{*}\right) X \mid Y\right) \\
= & \operatorname{Re}(A Z \mid Z)-\left(1-\frac{4}{p p^{\prime}}\right) \operatorname{Re}(A X \mid X) \\
& +\operatorname{Im}\left(\left(\frac{2}{p}-1\right) A X+\left(\frac{2}{p^{\prime}}-1\right) A^{*} X \mid Y\right) .
\end{aligned}
$$


As we have $1-4 /\left(p p^{\prime}\right)=(1-2 / p)^{2}$ and $2 / p^{\prime}-1=1-2 / p$, the right-hand side of $(9)$ is precisely what we have to bound from below. By definition, the left-hand side of (9) is a.e. on $O$ greater than or equal to

$$
\frac{1}{2} \Delta_{p}(A)|\widetilde{Z}|^{2}=\Delta_{p}(A)\left(\frac{2}{p}|X|^{2}+\frac{p}{2}|Y|^{2}\right) .
$$

The following calculation is similar to [11, Lem. 2.5] and, in fact, also lies at the heart of the matter in [5].

Lemma 10. Let $p \in[2, \infty)$. Given $u \in V$ and $n \in \mathbb{N}$, define

$$
v=u\left(|u|^{p / 2-1} \wedge n\right), \quad w=u\left(|u|^{p-2} \wedge n^{2}\right)
$$

as well as

$$
\chi=\mathbf{1}_{\left[|u|^{p-2} \geq n^{2}\right]}, \quad \chi_{c}=\mathbf{1}_{\left[|u|^{p-2}<n^{2}\right]} .
$$

Then $v, w \in V$ and, abbreviating $Z=\overline{\operatorname{sgn} v} \nabla v, X=\operatorname{Re} Z$, and $Y=\operatorname{Im} Z$, it follows that

$$
\begin{aligned}
& \overline{\operatorname{sgn} v} \nabla u=\frac{1}{n} \chi Z+\chi_{c}|v|^{2 / p-1}\left(Z-\left(1-\frac{2}{p}\right) X\right), \\
& \overline{\operatorname{sgn} v} \nabla w=n \chi Z+\chi_{c}|v|^{1-2 / p}\left(Z+\left(1-\frac{2}{p}\right) X\right) .
\end{aligned}
$$

Proof. The functions $z \mapsto z\left(|z|^{p / 2-1} \wedge n\right)$ and $z \mapsto z\left(|z|^{p-2} \wedge n^{2}\right)$ are Lipschitz continuous on $\mathbb{C}$ and vanish for $z=0$. Lemma 4.(i) asserts $v, w \in V$.

Explicit formulæ for their gradient are derived from the product rule and then the chain rule applied to the second factor that only involves the real-valued function $|u|$. (If $p<3$, some additional smoothing is required since $|\cdot|^{p-2}$ fails to be Lipschitz about 0 .) - Details of this argument have carefully been written down in [22, Lemma 5.2] and the result is as expected:

$$
\begin{aligned}
& \nabla v=n \chi \nabla u+\chi_{c}|u|^{p / 2-1}\left(\nabla u+\left(\frac{p}{2}-1\right)(\operatorname{sgn} u) \nabla|u|\right) \\
& \nabla w=n^{2} \chi \nabla u+\chi_{c}|u|^{p-2}(\nabla u+(p-2)(\operatorname{sgn} u) \nabla|u|) .
\end{aligned}
$$

Since $|v|$ is obtained from $|u| \in \mathrm{W}^{1,2}(O)$ in the same way as $v$ is obtained from $u$, we have

$$
\nabla|v|=n \chi \nabla|u|+\chi_{c} \frac{p}{2}|u|^{p / 2-1} \nabla|u| .
$$

We multiply this equation by $\chi_{c}(1-2 / p)$ to find

$$
\chi_{c}\left(1-\frac{2}{p}\right) \nabla|v|=\chi_{c}\left(\frac{p}{2}-1\right)|u|^{p / 2-1} \nabla|u| .
$$

Together with the identities $\chi_{c}|v|=\chi_{c}|u|^{p / 2}$ and $\chi_{c} \operatorname{sgn}(u)=\chi_{c} \operatorname{sgn}(v)$, which follow right away from the definition of $v$, the previous identity allows us to solve (10) for

$$
\nabla u=\frac{1}{n} \chi \nabla v+\chi_{c}|v|^{2 / p-1}\left(\nabla v-\left(1-\frac{2}{p}\right)(\operatorname{sgn} v) \nabla|v|\right) .
$$


We use the same identities to rewrite (11) as

$$
\nabla w=n^{2} \chi \nabla u+\chi_{c}\left(|v|^{2-4 / p} \nabla u+\left(2-\frac{4}{p}\right)|v|^{1-2 / p}(\operatorname{sgn} v) \nabla|v|\right)
$$

and plug in the right-hand side of (12) for $\nabla u$ to give

$$
\nabla w=n \chi \nabla v+\chi_{c}|v|^{1-2 / p}\left(\nabla v+\left(1-\frac{2}{p}\right)(\operatorname{sgn} v) \nabla|v|\right)
$$

In order to conclude, it suffices to multiply (12) and (13) by $\overline{\operatorname{sgn} v}$ each, on recalling $\nabla|v|=\operatorname{Re}(\overline{\operatorname{sgn} v} \nabla v)=X$ and $\operatorname{sgn} v \overline{\operatorname{sgn} v}=1$ on the set where $v \neq 0$.

We combine the previous two lemmata in order to establish the formal $\mathrm{L}^{p}$-dissipativity of $L$. It will become important that we do not assume $u|u|^{p / 2-1} \in \mathrm{W}_{\text {loc }}^{1,2}(O)$ a priori as in $[8$, Thm. 2.4].

Proposition 11. Let $p \in[2, \infty)$. If $A$ is $p$-elliptic and $u \in \mathcal{D}(L) \cap \mathrm{L}^{p}(O)$ is such that $L u \in \mathrm{L}^{p}(O)$, then it follows that $u|u|^{p / 2-1} \in V$ and

$$
\operatorname{Re}\left(\left.L u|u| u\right|^{p-2}\right) \geq \frac{2 \Delta_{p}(A)}{p}\left\|\nabla\left(u|u|^{p / 2-1}\right)\right\|_{2}^{2}
$$

Proof. Let $v, w$ be defined as in Lemma 10 for some $n \in \mathbb{N}$ that we shall not need to specify at this stage of the proof. We have $v, w \in V$ and in particular

$$
\operatorname{Re}(L u \mid w)=\operatorname{Re} a(u, w)=\int_{O} \operatorname{Re}(A(x) \nabla u(x) \mid \nabla w(x)) \mathrm{d} x .
$$

We shall derive a lower bound for the integrand that holds a.e. on $O$. We have $\operatorname{sgn} v=$ $\operatorname{sgn}(u)$, which in turn implies $\operatorname{sgn} v \overline{\operatorname{sgn} v} \nabla u=\nabla u$. Relying on the notation of Lemma 10, we first write

$$
(A \nabla u \mid \nabla w)=(A \overline{\operatorname{sgn} v} \nabla u \mid \overline{\operatorname{sgn} v} \nabla w) .
$$

Then we insert the two formulæ provided by Lemma 10 on the right, taking into account $\chi \chi_{c}=0$ and that the scalar term $|v|^{1-2 / p}$ commutes with $A$, to give

$$
(A \nabla u \mid \nabla w)=\chi(A Z \mid Z)+\chi_{c}\left(A Z-\left(1-\frac{2}{p}\right) A X \mid Z+\left(1-\frac{2}{p}\right) X\right) .
$$

Taking real parts and simplifying $\left(\chi+\chi_{c}=1\right)$, we arrive at

$\operatorname{Re}(A \nabla u \mid \nabla w)=\operatorname{Re}(A Z \mid Z)-\chi_{c}\left(1-\frac{2}{p}\right)^{2} \operatorname{Re}(A X \mid X)-\chi_{c}\left(1-\frac{2}{p}\right) \operatorname{Im}\left(\left(A-A^{*}\right) X \mid Y\right)$.

Now, we can use Lemma 9 and then standard ellipticity of $A$ to bound the right-hand side from below in order to deduce

$$
\begin{aligned}
\operatorname{Re}(A \nabla u \mid \nabla w) & \geq \chi \operatorname{Re}(A Z \mid Z)+\chi_{c} \Delta_{p}(A)\left(\frac{2}{p}|X|^{2}+\frac{p}{2}|Y|^{2}\right) \\
& \geq \chi \lambda|Z|^{2}+\chi_{c} \frac{2 \Delta_{p}(A)}{p}|Z|^{2}
\end{aligned}
$$


where in the final step we have also used that $p \geq 2$. We employ this estimate along with $|Z|=|\nabla v|$ on the right-hand side of (14) to obtain

$$
\operatorname{Re}(L u \mid w) \geq \int_{O} \chi \lambda|\nabla v|^{2}+\chi_{c} \frac{2 \Delta_{p}(A)}{p}|\nabla v|^{2} \mathrm{~d} x .
$$

At this stage, we prefer to write $v^{(n)}, w^{(n)}, \chi^{(n)}$, and $\chi_{c}^{(n)}$, since they all depend on the level of truncation $n$. In the limit as $n \rightarrow \infty$, we obtain from Lebesgue's dominated convergence that $w^{(n)} \rightarrow u|u|^{p-2}$ in $\mathrm{L}^{p^{\prime}}$ norm and $v^{(n)} \rightarrow u|u|^{p / 2-1}$ in $\mathrm{L}^{2}$ norm. Taking into account $\chi^{(n)}+\chi_{c}^{(n)}=1$, we get from (16) the rough bound

$$
\operatorname{Re}\left(L u \mid w^{(n)}\right) \geq\left(\lambda \wedge \frac{2 \Delta_{p}(A)}{p}\right)\left\|\nabla v^{(n)}\right\|_{2}^{2},
$$

which entails a uniform bound for $\nabla v^{(n)}$ in $\mathrm{L}^{2}(O)$. Altogether, we have shown that $\left(v^{(n)}\right)_{n}$ is uniformly bounded in $V$ and thus admits a subsequence with weak limit $v^{(\infty)} \in V$. But then we must have $v^{(\infty)}=u|u|^{p / 2-1}$ and consequently $u|u|^{p / 2-1} \in V$.

Next, we show pointwise convergence $\nabla v^{(n)} \rightarrow \nabla\left(u|u|^{p / 2-1}\right)$ a.e. on $O$. Every function in $\mathrm{W}^{1,2}(O)$ admits a representative that is absolutely continuous on almost all line segments in $O$ parallel to the coordinate axes and whose classical derivatives are representatives for the weak derivatives in the respective direction. See [24, Thm. 2.1.4] for this so-called Beppo Levi Property. On a segment parallel to a coordinate axis $x_{i}$ with this property for $u$, we can use the classical chain rule for the composition of $u$ with the $\mathrm{C}^{1}$ function $t \mapsto t|t|^{p / 2-1}$ to compute $\partial_{x_{i}}\left(u|u|^{p / 2-1}\right)=|u|^{p / 2-1}\left(\partial_{x_{i}} u+(p / 2-1) \operatorname{sgn}(u) \partial_{x_{i}}|u|\right)$. Since every such segment also admits the property for $u|u|^{p / 2-1}$, we conclude

$$
\nabla\left(u|u|^{p / 2-1}\right)=|u|^{p / 2-1}(\nabla u+(p / 2-1) \operatorname{sgn}(u) \nabla|u|)
$$

on $O$ in the weak sense. Due to (10), the right-hand side above is the pointwise limit of $\nabla v^{(n)}$ as $n \rightarrow \infty$ a.e. on $O$.

Eventually, we can pass to the limit in (16) via Fatou's lemma to conclude

$$
\begin{aligned}
\operatorname{Re}\left(\left.L u|u| u\right|^{p-2}\right) & =\lim _{n \rightarrow \infty} \operatorname{Re}\left(L u \mid w^{(n)}\right) \\
& \geq \int_{O} \liminf _{n \rightarrow \infty}\left(\chi^{(n)} \lambda\left|\nabla v^{(n)}\right|^{2}+\chi_{c}^{(n)} \frac{2 \Delta_{p}(A)}{p}\left|\nabla v^{(n)}\right|^{2}\right) \mathrm{d} x \\
& =\frac{2 \Delta_{p}(A)}{p}\left\|\nabla\left(u|u|^{p / 2-1}\right)\right\|_{2}^{2} .
\end{aligned}
$$

In fact, Proposition 11 will only be used in the next section, whereas here we need a slight variant that also applies when $\Delta_{p}(A)=0$. For pure Dirichlet conditions, such variant was obtained in [4, Prop. 5.8 \& Prop. 7.6] and their proofs could also be adapted.

Corollary 12. Let $p \in[2, \infty)$ and suppose $\Delta_{p}(A) \geq 0$. If $u \in V$ is such that $u|u|^{p-2} \in V$, then $\operatorname{Re} a\left(u, u|u|^{p-2}\right) \geq 0$.

Proof. We repeat the main steps of the proof of Proposition 11 and use the same notation. Without any assumption but $u \in V$, we have again (15). Hence, $\operatorname{Re}\left(A \nabla u \mid \nabla w^{(n)}\right) \geq 0$ holds a.e. on $O$. Since we assume $u|u|^{p-2} \in \mathrm{W}^{1,2}(O)$, we can use again the Beppo Levi 
Property and (11) to conclude $\nabla w^{(n)} \rightarrow \nabla\left(u|u|^{p-2}\right)$ a.e. on $O$ as $n \rightarrow \infty$. This implies $\operatorname{Re}\left(A \nabla u \mid \nabla\left(u|u|^{p-2}\right)\right) \geq 0$ a.e. on $O$, whereupon the claim follows by integration.

The link between formal $\mathrm{L}^{p}$-dissipativity as in Corollary 12 and $\mathrm{L}^{p}$-contractivity of the semigroup generated by $-L$ is provided by a beautiful result of Nittka [19].

We denote by $P_{B^{p}}$ the orthogonal projection of $\mathrm{L}^{2}(O)$ onto the $\mathrm{L}^{p}$ unit ball $B^{p}:=\{u \in$ $\left.\mathrm{L}^{2}(O) \cap \mathrm{L}^{p}(O):\|u\|_{p} \leq 1\right\}$. This is a convex and closed subset of $\mathrm{L}^{2}(O)$. According to [19, Theorem 4.1], the following assertions are equivalent:

(i) $V$ is invariant under $P_{B^{p}}$, and $\operatorname{Re} a\left(u, u|u|^{p-2}\right) \geq 0$ for every $u \in V$ satisfying $u|u|^{p-2} \in V$.

(ii) $\|T(t) f\|_{p} \leq\|f\|_{p}$ for all $f \in \mathrm{L}^{2}(O) \cap \mathrm{L}^{p}(O)$ and all $t \geq 0$.

Moreover, given $f \in \mathrm{L}^{2}(O) \backslash B^{p}$ we have for $u:=P_{B^{p}} f$ the implicit formula

$$
f=u+t u|u|^{p-2}
$$

for a constant $t>0$ depending on $f$ and $u$, see [19, Thm. 3.3(a)].

Proposition 13. If $\Delta_{p}(A) \geq 0$, then $\|T(t) f\|_{p} \leq\|f\|_{p}$ holds for all $f \in \mathrm{L}^{2}(O) \cap \mathrm{L}^{p}(O)$ and all $t \geq 0$.

Proof. By duality, it suffices to treat the case $p \geq 2$, see Lemma 8 and [18, Thm. VI.2.5] for duality theory for $L$. Of course we intend to use Nittka's result and verify (i). The second part is precisely the statement of Corollary 12. The invariance part is obvious for $f \in V \cap B^{p}$ since $P_{B^{p}}$ is a projection. Hence, we can focus on $f \in V \backslash B^{p}$, in which case we have the implicit formula (17).

We consider the function $\Upsilon: \mathbb{R} \rightarrow \mathbb{R}$ defined by $\Upsilon(s)=s+t s|s|^{p-2}$, which is strictly increasing, vanishes at 0 , and is continuously differentiable with derivative $\Upsilon^{\prime}(s)=1+$ $t(p-1)|s|^{p-2} \geq 1$. This implies that $\Psi:=\Upsilon^{-1}$ is continuously differentiable, strictly increasing and that it satisfies $\left\|\Psi^{\prime}\right\|_{\infty} \leq 1, \Psi^{\prime}(0)=1$, and $\Psi(0)=0$. We take absolute values in (17) to find $|f|=\Upsilon(|u|)$, that is to say, $|u|=\Psi(|f|)$. Therefore, we can rewrite $(17)$ as $u=\Phi(f)$, with $\Phi: \mathbb{C} \rightarrow \mathbb{C}$ given by

$$
\Phi(z)=\frac{z}{1+t \Psi(|z|)^{p-2}} .
$$

We identify $\mathbb{C} \cong \mathbb{R}^{2}$ and write $z=x+\mathrm{i} y$ with $x, y \in \mathbb{R}$. Clearly, $\Phi$ vanishes at $z=0$ and has continuous partial derivatives in every $z \neq 0$. If for $|z|$ close to 0 and $\infty$ we can bound those uniformly, then $\Phi$ will be Lipschitz continuous and $u \in V$ will follow from Lemma 4.

To this end, we begin with a direct calculation for $z \neq 0$ leading to

$$
\begin{aligned}
\frac{1}{2}\left(\left|\partial_{x} \Phi(z)\right|+\left|\partial_{y} \Phi(z)\right|\right) & \leq \frac{1}{1+t \Psi(|z|)^{p-2}}+\frac{t(p-2)|z| \Psi(|z|)^{p-3} \Psi^{\prime}(|z|)}{\left(1+t \Psi(|z|)^{p-2}\right)^{2}} \\
& \leq 1+t(p-2) \frac{|z| \Psi(|z|)^{p-3}}{\left(1+t \Psi(|z|)^{p-2}\right)^{2}}
\end{aligned}
$$


where we have used $\Psi(|z|) \geq 0$ and $\Psi^{\prime}(|z|) \leq 1$ in the second step. For $s \geq 1$ we obtain $\Upsilon(s) \leq(1+t) s^{p-1}$, which entails $|z| \leq(1+t) \Psi(|z|)^{p-1}$ for $|z| \geq \Upsilon(1)$. In this case we have

$$
\frac{1}{2}\left(\left|\partial_{x} \Phi(z)\right|+\left|\partial_{y} \Phi(z)\right|\right) \leq 1+\frac{(p-2)|z|}{t \Psi(|z|)^{p-1}} \leq 1+\frac{(p-2)(1+t)}{t} .
$$

For $|z|$ sufficiently small, we have $|\Psi(z)| \approx|z|$ thanks to $\Psi(0)=0$ and $\Psi^{\prime}(0)=1$ and thus

$$
\frac{1}{2}\left(\left|\partial_{x} \Phi(z)\right|+\left|\partial_{y} \Phi(z)\right|\right) \lesssim 1+|z|^{p-2}
$$

Theorem 2 follows from Proposition 13 by a routine interpolation argument.

Proof of Theorem 2. The first part follows from Proposition 13, Lemma 8, and the semigroup properties on $\mathrm{L}^{2}(O)$ by means of Stein's interpolation theorem. The reader can refer to [21, p. 96] or [2, Thm. 10.8] for this argument. Extrapolation up to $q=p$ or $q=p^{\prime}$ under the stronger assumption that $A$ is $p$-elliptic comes for free since the latter is an open-ended condition in $p$, see Section 1.2.

\section{Extrapolation to A HOLOMORPhic SEMigroup}

We turn toward the proof of Theorem 1 . We recall that $\pi / 2-\omega$ is the angle of holomorphy of the semigroup $T$ generated by $-L$ on $\mathrm{L}^{2}(O)$.

Definition 14. Let $\psi \in[0, \pi)$. Given $p, q \in(1, \infty)$ with $p \leq q$, a family of operators $(S(z))_{z \in \mathrm{S}_{\psi}^{+}} \subseteq \mathcal{L}\left(\mathrm{L}^{2}(O)\right)$ is said to be $p \rightarrow q$ bounded if

$$
\|S(z) f\|_{q} \leq C|z|^{d /(2 q)-d /(2 p)}\|f\|_{p}
$$

holds for some constant $C$ and all $z \in \mathrm{S}_{\psi}^{+}$and all $f \in \mathrm{L}^{p}(O) \cap \mathrm{L}^{2}(O)$.

We start out with a Nash-type inequality.

Lemma 15. Assume $d \geq 3$ and that $V$ has the embedding property. Suppose that $(T(t))_{t>0}$ is $p \rightarrow p$ bounded and let $\varepsilon>0$. If $p<2$, then the shifted semigroup $\left(\mathrm{e}^{-\varepsilon t} T(t)\right)_{t>0}$ is $p \rightarrow 2$ bounded and if $p>2$, then it is $2 \rightarrow p$ bounded. If $V$ has the homogeneous embedding property, then the conclusion also holds for $\varepsilon=0$.

Proof. By duality it suffices to treat the case $p<2$. For $v \in V$, we obtain from Hölder's inequality and the embedding property

$$
\|v\|_{2} \leq\|v\|_{p}^{1-\theta}\|v\|_{2^{*}}^{\theta} \lesssim\|v\|_{p}^{1-\theta}\|v\|_{1,2}^{\theta}
$$

where $\theta \in(0,1)$ is such that $1 / 2=(1-\theta) / p+\theta / 2^{*}$. Given $f \in \mathrm{L}^{2}(O) \cap \mathrm{L}^{p}(O)$ with $\|f\|_{p}=1$, we set $v(t):=\mathrm{e}^{-\varepsilon t} T(t) f$ for $t>0$. Ellipticity yields

$$
\|v(t)\|_{1,2}^{2} \lesssim \operatorname{Re} a(v(t), v(t))+\varepsilon(v(t) \mid v(t))=\operatorname{Re}((L+\varepsilon) v(t) \mid v(t))=-\frac{1}{2} \frac{\mathrm{d}}{\mathrm{d} t}\|v(t)\|_{2}^{2} .
$$


By assumption, we have $\|v(t)\|_{p} \lesssim \mathrm{e}^{-\varepsilon t}\|f\|_{p} \leq 1$. Combining these three estimates leads us to a differential inequality for $t \mapsto\|v(t)\|_{2}^{2}$ :

$$
\|v(t)\|_{2}^{2 / \theta} \leq-C\left(\|v(t)\|_{2}^{2}\right)^{\prime}
$$

where $C>0$ depends on the assumptions and ellipticity. If $V$ has the homogeneous embedding property, then we replace $\|v\|_{1,2}$ by $\|\nabla v\|_{2}$ in the first line, and hence we can take $\varepsilon=0$.

If $v$ vanishes somewhere on $(t / 2, t)$, then $v(t)=0$ by the semigroup property and we are done. Otherwise, we obtain

$$
\frac{t}{2} \leq-\int_{t / 2}^{t} \frac{C\left(\|v(s)\|_{2}^{2}\right)^{\prime}}{\|v(s)\|_{2}^{2 / \theta}} \mathrm{d} s \leq \frac{C \theta}{1-\theta}\|v(t)\|_{2}^{2-2 / \theta}=\frac{C \theta}{1-\theta}\|v(t)\|_{2}^{-4 p /(2 d-p d)},
$$

which is the required $p \rightarrow 2$ estimate.

The following lemma is a blend of ideas found in [3, Sec. 3]. It plays a crucial role for both extrapolating the range of exponents from Theorem 2 and obtaining the optimal angle of holomorphy.

Lemma 16. Let $\varepsilon \geq 0$ and suppose either $p<2$ and that $\left(\mathrm{e}^{-\varepsilon t} T(t)\right)_{t>0}$ is $p \rightarrow 2$ bounded or suppose $p>2$ and that it is $2 \rightarrow p$ bounded. Then for every $\psi \in[0, \pi / 2-\omega)$ and every $q$ between 2 and $p$ the holomorphic extension $\left(\mathrm{e}^{-\varepsilon z} T(z)\right)_{z \in \mathrm{S}_{\psi}^{+}}$is $q \rightarrow q$ bounded.

Proof. As usual we invoke a duality argument and confine ourselves this time to $p<2$. We fix some angle $\phi \in(\psi, \pi / 2-\omega)$. Every $z \in \mathrm{S}_{\psi}^{+}$can be written as $z=z^{\prime}+t$, where $z^{\prime} \in \mathrm{S}_{\phi}^{+}$and $t>0$ satisfy $|z| \approx\left|z^{\prime}\right| \approx t$ with implied constants depending on $\phi$ and $\psi$. By the semigroup law, we find

$$
\left\|\mathrm{e}^{-\varepsilon z} T(z)\right\|_{p \rightarrow 2} \leq\left\|\mathrm{e}^{-\varepsilon z^{\prime}} T\left(z^{\prime}\right)\right\|_{2 \rightarrow 2}\left\|\mathrm{e}^{-\varepsilon t} T(t)\right\|_{p \rightarrow 2} \lesssim t^{d / 4-d /(2 p)} \approx|z|^{d / 4-d /(2 p)} .
$$

In particular, for all measurable sets $E, F \subseteq O$ and all $f \in \mathrm{L}^{2}(O) \cap \mathrm{L}^{p}(O)$ with support in $E$ we have

$$
\left\|\mathrm{e}^{-\varepsilon z} T(z) f\right\|_{\mathrm{L}^{2}(F)} \leq|z|^{d / 4-d /(2 p)}\|f\|_{\mathrm{L}^{p}(E)} .
$$

Riesz-Thorin interpolation with the off-diagonal bound provided by Proposition 7 yields for all $f \in \mathrm{L}^{2}(O) \cap \mathrm{L}^{q}(O)$ with support in $E$ the estimate

$$
\left\|\mathrm{e}^{-\varepsilon z} T(z) f\right\|_{\mathrm{L}^{2}(F)} \lesssim|z|^{d / 4-d /(2 q)} \mathrm{e}^{-\frac{\theta \mathrm{d}(E, F)^{2}}{4 C|z|}}\|f\|_{\mathrm{L}^{q}(E)}
$$

where $\theta \in(0,1)$ satisfies $1 / q=(1-\theta) / p+\theta / 2$. This implies $q \rightarrow q$ boundedness thanks to the subsequent lemma applied with $g(r)=|z|^{d / 4-d /(2 q)} \mathrm{e}^{-\frac{\theta r^{2}}{4 C|z|}}$ and $s=\sqrt{|z|}$.

We cite $[9$, Lem. 4.5$]$ with slight change in notation to link it with the above proof.

Lemma 17. Let $1 \leq q \leq 2$ and $S$ a bounded linear operator on $\mathrm{L}^{2}(O)$. Assume that $S$ satisfies off-diagonal estimates in the form

$$
\|S f\|_{\mathrm{L}^{2}(R \cap O)} \leq g(\mathrm{~d}(Q, R))\|f\|_{\mathrm{L}^{q}(Q \cap O)},
$$


whenever $Q, R$ are closed axis-parallel cubes in $\mathbb{R}^{d}, f \in \mathrm{L}^{2}(O) \cap \mathrm{L}^{q}(O)$ is supported in $Q \cap O$, and $g$ is some decreasing function. Then $S$ is $q \rightarrow q$ bounded with norm bounded by $s^{d / 2-d / q} \sum_{k \in \mathbb{Z}^{d}} g(s \max \{|k| / \sqrt{d}-1,0\})$ for any $s>0$ provided this sum is finite.

The next proposition yields Theorem 1 up to formalities that we discuss afterward.

Proposition 18. Assume $d \geq 3$ and that $V$ has the embedding property. Let $p>2$ and assume that $A$ is p-elliptic. Then for every $\psi \in[0, \pi / 2-\omega)$ and every $\varepsilon>0$ the semigroup $\left(\mathrm{e}^{-\varepsilon z} T(z)\right)_{z \in \mathrm{S}_{\psi}^{+}}$is $q \rightarrow q$ bounded for $q \in(2, d p /(d-2))$. If $V$ has the homogeneous embedding property, then the same result holds for $\varepsilon=0$.

Proof. Let $u \in \mathcal{D}(L) \cap \mathrm{L}^{p}(O)$ such that $L u \in \mathrm{L}^{p}(O)$. According to Proposition 11, we have $v:=u|u|^{p / 2-1} \in V$ along with the bound

$$
\frac{2 \Delta_{p}(A)}{p}\|\nabla v\|_{2}^{2} \leq \operatorname{Re}\left(\left.L u|u| u\right|^{p-2}\right)
$$

Hence, we get

$$
\frac{2 \Delta_{p}(A)}{p}\|\nabla v\|_{2}^{2}+\|v\|_{2}^{2} \leq \operatorname{Re}\left(\left.(1+L) u|u| u\right|^{p-2}\right) .
$$

We use the embedding property of $V$ on the left and Hölder's inequality on the right to give $\|v\|_{2^{*}}^{2} \lesssim\|(1+L) u\|_{p}\left\|u|u|^{p-2}\right\|_{p^{\prime}}$. By definition of $v$, this can be rewritten as

$$
\|u\|_{r} \lesssim\|(1+L) u\|_{p}^{1 / p}\|u\|_{p}^{1 / p^{\prime}} \leq\left(\|u\|_{p}+\|L u\|_{p}\right)^{1 / p}\|u\|_{p}^{1 / p^{\prime}},
$$

where $r=d p /(d-2)$ and the second step is just the triangle inequality.

Now, let $f \in \mathrm{L}^{2}(O) \cap \mathrm{L}^{p}(O)$ and $t>0$. We intend to apply the previous estimate to $u:=T(t) f$. Indeed, we have $u \in \mathcal{D}(L)$ by holomorphy of the semigroup on $\mathrm{L}^{2}(O)$. Combining Theorem 2 and Lemma 15 leads us to

$$
\|u\|_{p} \lesssim \mathrm{e}^{\varepsilon t / 2} t^{d /(2 p)-d / 4}\|f\|_{2},
$$

where $\varepsilon>0$ is arbitrary but the implied constant depends on $\varepsilon$. The same argument together with holomorphy of the semigroup on $\mathrm{L}^{2}(O)$ yields

$$
\|L u\|_{p}=\|T(t / 2) L T(t / 2) f\|_{p} \lesssim \mathrm{e}^{\varepsilon t / 2} t^{d /(2 p)-d / 4}\|L T(t / 2) f\|_{2} \lesssim \mathrm{e}^{\varepsilon t / 2} t^{d /(2 p)-d / 4-1}\|f\|_{2} .
$$

We use these two bounds on the right hand side of (19) to give

$$
\|T(t) f\|_{r} \lesssim \mathrm{e}^{\varepsilon t / 2} t^{d /(2 p)-d / 4-1 / p}(1+t)^{1 / p}\|f\|_{2} .
$$

Now, we multiply by $\mathrm{e}^{-\varepsilon t}$ and invoke uniform boundedness of $(1+t)^{1 / p} \mathrm{e}^{-\varepsilon t / 2}$ for $t>0$ to ultimately obtain

$$
\left\|\mathrm{e}^{-\varepsilon t} T(t) f\right\|_{r} \lesssim t^{d /(2 p)-d / 4-1 / p}\|f\|_{2} .
$$

As $r=d p /(d-2)$, the exponent of $t$ on the right just happens to be $d /(2 r)-d / 4$, so that the above estimate proves $2 \rightarrow r$ boundedness of $\left(\mathrm{e}^{-\varepsilon t} T(t)\right)_{t>0}$. We conclude from Lemma 16 that for every $\psi \in[0, \pi / 2-\omega)$ and every $q \in(2, r)$ the holomorphic extension $\left(\mathrm{e}^{-\varepsilon z} T(z)\right)_{z \in S_{\psi}^{+}}$is $q \rightarrow q$ bounded as required. 
The modifications if $V$ even has the homogeneous embedding property are straightforward. In this case we can skip (18) and directly obtain $\|u\|_{r} \lesssim\|L u\|_{p}^{1 / p}\|u\|_{p}^{1 / p^{\prime}}$ as replacement for (19). From thereon, we can proceed as before, the only exceptions being that we take $\varepsilon=0$ in Lemmas 15 and 16 and that the factor $(1+t)^{1 / p}$ no longer shows up in $(20)$.

Finally, we give the

Proof of Theorem 1. By duality and Lemma 8, it suffices to treat the case $p, q>2$. For such $q$ verifying

$$
|1 / 2-1 / q|<1 / d+(1-2 / d)|1 / 2-1 / p|
$$

the conclusion follows from Proposition 18 and holomorphy of the semigroup on $\mathrm{L}^{2}(O)$ just as in proof Theorem 2. Indeed, we can use Stein interpolation for the restriction of $T$ to any ray $[0, \infty) \mathrm{e}^{ \pm \mathrm{i} \psi}$ for $\psi \in[0, \pi / 2-\omega)$. The endpoint case for $q$ is again for free since $p$-ellipticity is an open-ended condition.

\section{APPENDIX: An EQUIVALENT FORMULATION OF $p$-ELLIPTICITY}

We provide a direct proof of a statement from [4, Prop. 5.15] that we have alluded to in Section 1.2.

Proposition A. Let $p \in(1, \infty)$ and suppose that $A: O \rightarrow \mathcal{L}\left(\mathbb{C}^{d}\right)$ satisfies (1). Then $\Delta_{p}(A)>0$ holds if and only if

$$
\mu(A):=\operatorname{essinf}_{x \in O} \inf _{\xi \in \mathbb{C}^{d},(A(x) \xi \mid \bar{\xi}) \neq 0} \frac{\operatorname{Re}(A(x) \xi \mid \xi)}{|(A(x) \xi \mid \bar{\xi})|}>|1-2 / p| .
$$

Proof. Let $\xi=\alpha+\mathrm{i} \beta \in \mathbb{C}^{d}$, where $\alpha, \beta \in \mathbb{R}^{d}$. By definition, we have

$$
\mathcal{J}_{p} \xi=2\left(\frac{\alpha}{p^{\prime}}+\frac{\mathrm{i} \beta}{p}\right)=\xi+\left(1-\frac{2}{p}\right) \bar{\xi}
$$

and

$$
\Delta_{p}(A)=\underset{x \in O}{\operatorname{essinf}} \min _{|\xi|=1} \operatorname{Re}((A(x) \xi \mid \xi)+(1-2 / p)(A(x) \xi \mid \bar{\xi})) .
$$

Since the set $\left\{\xi \in \mathbb{C}^{d}:|\xi|=1\right\}$ is preserved through multiplication by any $\omega \in \mathbb{C}$ of modulus 1 , we may replace $\xi$ by $\xi \omega$ and then take the minimum over $\xi$ and $\omega$ to give

$$
\Delta_{p}(A)=\underset{x \in O}{\operatorname{essinf}} \min _{|\xi|=1} \min _{|\omega|=1} \operatorname{Re}\left((A(x) \xi \mid \xi)+\omega^{2}(1-2 / p)(A(x) \xi \mid \bar{\xi})\right) .
$$

Given $x$ and $\xi$, the minimum over $\omega$ is attained precisely when

$$
\omega^{2}(1-2 / p)(A(x) \xi \mid \bar{\xi})=-|1-2 / p||(A(x) \xi \mid \bar{\xi})|
$$

and we conclude that

$$
\Delta_{p}(A)=\operatorname{essinf}_{x \in O} \min _{|\xi|=1}(\operatorname{Re}(A(x) \xi \mid \xi)-|1-2 / p||(A(x) \xi \mid \bar{\xi})|) .
$$


Owing to (1) we have for a.e. $x \in O$ a uniform upper bound $|(A(x) \xi \mid \bar{\xi})| \leq \Lambda$ for all $\xi \in \mathbb{C}^{d}$. Hence, if $\Delta_{p}(A)>0$ holds, then so does

$$
\underset{x \in O}{\operatorname{essinf}} \inf _{|\xi|=1,(A(x) \xi \mid \bar{\xi}) \neq 0} \frac{\operatorname{Re}(A(x) \xi \mid \xi)-|1-2 / p||(A(x) \xi \mid \bar{\xi})|}{|(A(x) \xi \mid \bar{\xi})|}>0 .
$$

Since the function we are minimizing is $\mathbb{R}$-homogeneous of degree 0 in $\xi$, this precisely means $\mu(A)>|1-2 / p|$. Conversely, let us assume that $\mu(A)>|1-2 / p|$ holds. On passing to the inverse, this is the same as having

$$
\operatorname{essinf}_{x \in O} \inf _{\xi \in \mathbb{C}^{d},(A(x) \xi \mid \bar{\xi}) \neq 0} \frac{\operatorname{Re}(A(x) \xi \mid \xi)-|1-2 / p||(A(x) \xi \mid \bar{\xi})|}{|1-2 / p| \operatorname{Re}(A(x) \xi \mid \xi)}>0 .
$$

Due to (1), we have for a.e. $x \in O$ the uniform bound $|1-2 / p| \operatorname{Re}(A(x) \xi \mid \xi) \geq|1-2 / p| \lambda$ for all $\xi \in \mathbb{C}^{d}$. Hence, the latter condition implies that the right-hand side in (21) is strictly positive and the proof is complete.

\section{REFERENCES}

[1] W. AREndt and A.F.M. TER Elst. Gaussian estimates for second order elliptic operators with boundary conditions. J. Operator Theory 38 (1997), no. 1, 87-130.

[2] W. Arendt, R. Chill, S. Seifert, H. Vogt, and J. Voigt. Form Methods for Evolution Equations, and Applications. Lecture Notes of the 18th Internet Seminar on Evolution Equations, https://www .mat.tuhh.de/home/pbaasch/isem18/pdf/LectureNotes . pdf .

[3] P. Auscher. On necessary and sufficient conditions for $L^{p}$-estimates of Riesz transforms associated to elliptic operators on $\mathbb{R}^{n}$ and related estimates. Mem. Amer. Math. Soc. 186 (2007), no. 871.

[4] A. Carbonaro and O. Dragičević. Convexity of power functions and bilinear embedding for divergence-form operators with complex coefficients. To appear in J. Eur. Math. Soc. (JEMS).

[5] A. CialdeA and V. MAZ'YA. Criterion for the $L^{p}$-dissipativity of second order differential operators with complex coefficients. J. Math. Pures Appl. (9) 38 (2005), no. 8, 1067-1100.

[6] D. DANERs. A priori estimates for solutions to elliptic equations on non-smooth domains. Proc. R. Soc. Edinburgh Sect. A 132 (2002), no. 4, 793-813.

[7] E.B. DAviEs. Uniformly elliptic operators with measurable coefficients. J. Funct. Anal. 132 (1995), no. $1,141-169$.

[8] M. Dindoš and J. PiPher. Regularity theory for solutions to second order elliptic operators with complex coefficients and the $L^{p}$ Dirichlet problem. Adv. Math. 341 (2019), 255-298.

[9] M. EGERT. $L^{p}$-estimates for the square root of elliptic systems with mixed boundary conditions. J. Differential Equations 265 (2018), no. 4, 1279-1323.

[10] M. Egert, R. Haller-Dintelmann, and J. Rehberg. Hardy's inequality for functions vanishing on a part of the boundary. Potential Anal. 43 (2015), no. 1, 49-78.

[11] A.F.M. Ter Elst, V. Liskevich, Z. Sobol, and H. Vogt. On the $L^{p}$-theory of $C_{0}$-semigroups associated with second-order elliptic operators with complex singular coefficients. Proc. Lond. Math. Soc. (3) 115 (2017), no. 4, 693-724.

[12] A.F.M. ter Elst, R. Haller-Dintelmann, J. Rehberg, and P. Tolksdorf. On the L ${ }^{p}$-theory for second-order elliptic operators in divergence form with complex coefficients. Preprint (2019), https://arxiv.org/abs/1903.06692

[13] A.F.M. TER Elst and J. RehBerg. $L^{\infty}$-estimates for divergence operators on bad domains. Anal. Appl. (Singap.) 10 (2012), no. 2, 207-214.

[14] K.-J. Engel and R. NAgel. One-Parameter Semigroups for Linear Evolution Equations. Graduate Texts in Mathematics, vol. 194, Springer, New York, 2000.

[15] S. Hofmann, S. Mayboroda, and A. McIntosh. Second order elliptic operators with complex bounded measurable coefficients in $L^{p}$, Sobolev and Hardy spaces. Ann. Sci. Éc. Norm. Supér. (4) 44 (2011), no. 5, 723-800. 
[16] G. LeOni and M. Morini. Necessary and sufficient conditions for the chain rule in $W_{\text {loc }}^{1,1}\left(\mathbb{R}^{N} ; \mathbb{R}^{d}\right)$ and $\mathrm{BV}_{\mathrm{loc}}\left(\mathbb{R}^{N} ; \mathbb{R}^{d}\right)$. J. Eur. Math. Soc. (JEMS) 9 (2007), no. 2, 219-252.

[17] S. Mourou and M. Selmi. Quasi-L $L^{p}$-contractive analytic semigroups generated by elliptic operators with complex unbounded coefficients on arbitrary domains. Semigroup Forum 85 (2012), no. 1, 5-36.

[18] T. Kato. Perturbation Theory for Linear Operators. Classics in Mathematics, Springer, Berlin, 1995.

[19] R. NitTKA. Projections onto convex sets and $L^{p}$-quasi-contractivity of semigroups. Arch. Math. (Basel) 98 (2012), no. 4, 341-353.

[20] E.M. Ouhabaz. Gaussian upper bounds for heat kernels of second-order elliptic operators with complex coefficients on arbitrary domains. J. Operator Theory 51 (2004), no. 2, 335-360.

[21] E.M. Ouhabaz. Analysis of Heat Equations on Domains. London Mathematical Society Monographs Series, vol. 31, Princeton University Press, Princeton NJ, 2005.

[22] Z. Sobol and H. Vogt. On the $L_{p}$-theory of $C_{0}$-semigroups associated with second-order elliptic operators. I. J. Funct. Anal. 193 (2002), no. 1, 24-54.

[23] P. Tolksdorf. $\mathcal{R}$-sectoriality of higher-order elliptic systems on general bounded domains. J. Evol. Eq. (2018), no. 2, 323-349 .

[24] W.P. Ziemer. Weakly differentiable functions. Graduate Texts in Mathematics, vol. 120, Springer, New York, 1989.

Laboratoire de Mathématiques d'Orsay, Univ. Paris-Sud, CNRS, Université Paris-Saclay, 91405 Orsay, France

Email address: moritz.egert@math.u-psud.fr 